\title{
Numerical simulation of 3D violent free-surface flows by multi-resolution MPS method
}

\author{
Zhenyuan Tang $^{1}$ - Decheng Wan ${ }^{1} \cdot$ Gang Chen $^{1} \cdot$ Qing Xiao $^{2}$
}

Received: 17 December 2015 / Accepted: 13 June 2016 / Published online: 11 July 2016

(C) Springer International Publishing Switzerland 2016

\begin{abstract}
D violent free-surface flows are modelled by multi-resolution moving particle semi-implicit (MPS) method. Firstly, a 2D dam-break flow with an obstacle is performed. This shows that multi-resolution MPS can provide the integrated and clear shape of free surface as that by universally fine (Uni-Fine) with high-resolution particles in the entire domain. Then the multi-resolution MPS method is employed to carry out 3D violent free-surface flows, including dambreak and water entry. In the dam-break case, a relative rational pressure field can be provided and the predicted impact pressures are also compared with experimental data. In the $3 \mathrm{D}$ water entry case, the present method can capture complex phenomena including largely free-surface deformations and splashing. The penetration depth of the cylinder is also close to experimental data.
\end{abstract}

Keywords MPS (moving particle semi-implicit) $\cdot$ Multiresolution method . 3D violent free-surface flows . Dam-break · Water entry

\section{Introduction}

Moving particle semi-implicit (MPS) method is one of the meshless particle methods. It was originally introduced by

$凶$ Decheng Wan

dcwan@sjtu.edu.cn

1 State Key Laboratory of Ocean Engineering, School of Naval Architecture, Ocean and Civil Engineering, Shanghai Jiao Tong University, Collaborative Innovation Center for Advanced Ship and Deep-Sea Exploration, Shanghai 200240, China

2 Department of Naval Architecture, Ocean and Marine Engineering, University of Strathclyde, Glasgow, UK
(Koshizuka and Oka 1996; Koshizuka et al. 1998) for a truly incompressible fluid. Numerous 2D works have been reported in the literature (Khayyer and Gotoh 2008, 2013; Zhang and Wan 2012; Zhang et al. 2013, 2014a; Tang and Wan 2015; Tamai and Koshizuka 2014; Shakibaeinia and Jin 2011). Moreover, the MPS method is also applied for violent free-surface flows in naval architecture and ocean engineering (Tang et al. 2016a,b). However, its high computational cost may limit its further application to 3D free-surface flows due to its semi-implicit algorithm, and the corresponding computational efficiency for 3D applications is a severe problem.

Up to now, many MPS researchers have attempted to accelerate computations in the frame of MPS by employing GPU and multi-CPU parallel techniques. Hori et al. (2011) and Zhu et al. (2011) discussed the efficiency of GPU computations for 2D free-surface flows. Kakuda et al. (2012, 2013) investigated GPU-accelerating performance using both 2D and 3D dam-break. Ovaysi and Piri (2012) presented a multi-GPU acceleration based on modified moving particle semi-implicit (MMPS) method. Iribe et al. (2010) discussed the optimization algorithm to reduce communication time among cores based on particle decomposition. Duan and Chen (2015) compared the performance of six kinds of pressure Poisson equation (PPE) methods by their parallel MPS solver. Gotoh et al. (2009) developed a 3D parallelized CMPS solver adopting a static domain-decomposition strategy. Zhang et al. (2013) also implemented a 3D parallel particle solver based on improved MPS and message passing interface (MPI) libraries and further applied it to green water flows. The above works have proved the capability of GPU and multi-CPU parallel in a particle-based MPS method, where the acceleration computations can be implemented based on increasing the hardware resources. Another alternative tool to accelerate the computation is the spatially 
local refine technique, which can be considered as an indirect acceleration method. Until now, there have been numerous spatially local refine techniques in the frame of weakly compressible SPH method (Oger et al. 2006; Vacondio et al. 2013; Omidvar et al. 2013; Barcarolo et al. 2014) and they have also been applied to free-surface flows, such as bow breaking wave (Marrone et al. 2011) and dam-break (Vacondio et al. 2012). However, a few of the relative works on the MPS method have been studied (Yoon et al. 1999; Shibata et al. 2012; Tanaka et al. 2009). The variable kernel size technique (Yoon et al. 1999) was mainly employed to investigate the 2D bubble dynamics by Heo et al. (2002), Tian et al. (2009) and Chen et al. (2010). In addition, the overlapping particle (Shibata et al. 2012) and multi-resolution techniques (Tanaka et al. 2009) were proposed for 2D free-surface flows and the relative $3 \mathrm{D}$ applications are rarely reported.

The main objective of the present work is to simulate $3 \mathrm{D}$ violent free-surface flows by multi-resolution MPS, where the multi-resolution technique is embedded into our in-house solver MLParticle-SJTU based on improved MPS (Zhang and Wan 2012; Zhang et al. 2016). The paper is organized in the following way. Firstly, the improved MPS method and the multi-resolution technique are described, such as particle interaction models and cutoff radii for two different-size neighboring particles. Next, the motion computation equation is described briefly. Finally, the multi-resolution MPS is applied to 3D violent free-surface flows, including 3D dambreak and water entry of cylinder flows. The comparisons between the numerical results and experimental data are also made.

\section{MPS numerical models}

\subsection{Governing equations}

In the MPS method, the governing equations are the continuity and Navier-Stokes equations, which can read as

$\frac{1}{\rho} \frac{\mathrm{D} \rho}{\mathrm{D} t}=-\nabla \cdot \boldsymbol{V}=0$

$\frac{D \boldsymbol{V}}{\mathrm{D} t}=-\frac{1}{\rho} \nabla P+v \nabla^{2} \boldsymbol{V}+\boldsymbol{g}$,

where $\mathrm{D} / \mathrm{D} t$ denotes the substantial or material derivative, $\rho$ is the fluid density, $\boldsymbol{V}$ is the velocity vector, $P$ presents the pressure, $v$ is the kinematic viscosity, $\boldsymbol{g}$ is the gravitational acceleration vector, and $t$ is the time.

\subsection{Particle interaction models}

Kernel function is quite significant in the particle-based method. In the present work, we adopt an improved ker- nel function without singularity (Zhang and Wan 2012) as follows:

$W(r)= \begin{cases}\frac{r_{e}}{0.85 r+0.15 r_{e}}-1 & 0 \leq r<r_{e} \\ 0 & r_{e} \leq r\end{cases}$

where $r_{e}$ denotes the cutoff radius of the particle interaction domain, and it is similar to the treatment by Koshizuka and Oka (1996). In addition, particle number density (PND) is defined as follows:

$\langle n\rangle_{i}=\sum_{j \neq i} W\left(\left|\mathbf{r}_{j}-\mathbf{r}_{i}\right|\right)$

In the MLParticle-SJTU solver, all differential operators in Eqs. (1) and (2) can be formulated as follows (Koshizuka et al. 1998; Tanaka and Masunaga 2010; Yoon et al. 1999; Shakibaeinia and Jin 2012):

$$
\begin{aligned}
& \langle\nabla P\rangle_{i}=\frac{D}{n^{0}} \sum_{j \neq i} \frac{P_{j}+P_{i}}{\left|\mathbf{r}_{j}-\mathbf{r}_{i}\right|^{2}}\left(\mathbf{r}_{j}-\mathbf{r}_{i}\right) W\left(\left|\mathbf{r}_{j}-\mathbf{r}_{i}\right|\right) \\
& \langle\nabla \cdot \mathbf{V}\rangle_{i}=\frac{D}{n^{0}} \sum_{j \neq i} \frac{\left(\mathbf{V}_{j}-\mathbf{V}_{i}\right) \cdot\left(\mathbf{r}_{j}-\mathbf{r}_{i}\right)}{\left|\mathbf{r}_{j}-\mathbf{r}_{i}\right|^{2}} W\left(\left|\mathbf{r}_{j}-\mathbf{r}_{i}\right|\right), \\
& \left\langle\nabla^{2} \phi\right\rangle_{i}=\frac{2 D}{n^{0} \lambda_{i}} \sum_{j \neq i}\left(\phi_{j}-\phi_{i}\right) W\left(\left|\mathbf{r}_{j}-\mathbf{r}_{i}\right|\right) \\
& \lambda_{i}=\frac{\sum_{j \neq i} W\left(\left|\mathbf{r}_{j}-\mathbf{r}_{i}\right|\right)\left|\mathbf{r}_{j}-r_{i}\right|^{2}}{\sum_{j \neq i} W\left(\left|\mathbf{r}_{j}-\mathbf{r}_{i}\right|\right)}
\end{aligned}
$$

where $n^{0}, D, \boldsymbol{r}$ and $\phi$ are the constant particle number density, the number of space dimensions, the coordinate vector of fluid particle and a physical quantity, respectively. In the following section, the left hand side of pressure Poisson equation (PPE) is discretized by Eq. (7).

\subsection{Model of incompressibility}

The incompressibility condition is expressed by the combination of the constant particle number density condition and divergence-free condition (Tanaka and Masunaga 2010; Lee et al. 2011):

$$
\left\langle\nabla^{2} P^{k+1}\right\rangle_{i}=(1-\gamma) \frac{\rho}{\Delta t} \nabla \cdot \mathbf{V}_{i}^{*}-\gamma \frac{\rho}{\Delta t^{2}} \frac{\left\langle n^{k}\right\rangle_{i}-n^{0}}{n^{0}}
$$

where the superscripts $k$ and $k+1$ indicate the physical quantities in the previous and current time level, $\Delta \mathrm{t}$ denotes the calculation time step and $\gamma$ is a parameter. For uniform resolution particles, $\gamma=0.01$ is adopted here. In addition, $\gamma$ is smaller for interface particles than that for uniform resolution particles. 


\subsection{Time stepping}

In the improved MPS method, a projection algorithm is employed to decouple the pressure-velocity problem. There are two stages in each time step (Tanaka and Masunaga 2010): firstly, a temporal velocity is predicted based on viscous and gravity force,

$\Delta \mathbf{V}_{i}^{*}=\Delta t\left(\nu \nabla^{2} \mathbf{V}_{i}^{k}+\mathbf{g}\right)$,

$\mathbf{V}_{i}^{*}=\mathbf{V}_{i}^{k}+\Delta \mathbf{V}_{i}^{*}$.

Then, the particle number density $n_{i}^{k}$ (Eq. (4)) and velocity divergence $\nabla \cdot V_{i}^{*}$ (Eq. (6)) are calculated and the Poisson equation of pressure (Eq. (9)) is solved to obtain the pressure field.

Finally, the velocity based on the pressure obtained from PPE is updated:

$$
\begin{aligned}
& \mathbf{V}_{i}^{k+1}=\mathbf{V}_{i}^{*}-\Delta t \frac{1}{\rho} \nabla P^{k+1}, \\
& \mathbf{r}_{i}^{k+1}=\mathbf{r}_{i}^{k}+\Delta t \cdot \mathbf{V}_{i}^{k+1} .
\end{aligned}
$$

\subsection{Boundary conditions}

Numerous algorithms are developed to assess the freesurface particles for particle-based simulations on the basis of small particle number density (Koshizuka et al. 1998), the number of neighboring particles (Tanaka and Masunaga 2010), the virtual light source and virtual screen (Shibata et al. 2015), and the asymmetry of neighboring particles (Khayyer et al. 2009; Zhang and Wan 2012). Here, the detection method by Zhang and Wan (2012) is employed. The function based on the asymmetric arrangement of neighboring particles is defined in Eq. (14):

$\mathbf{F}_{i}=\frac{D}{n^{0}} \sum_{j \neq i} \frac{1}{\left|\mathbf{r}_{i}-\mathbf{r}_{j}\right|}\left(\mathbf{r}_{i}-\mathbf{r}_{j}\right) W\left(r_{i j}\right)$,

$\left|\mathbf{F}_{i}\right|>\alpha$, for free-surface particles,

where $\alpha=0.9|\boldsymbol{F}|^{0}$ and $|\boldsymbol{F}|^{0}$ is the initial value of the surface particle. $\boldsymbol{F}$ is a vector with a large absolute value for surface particles or particles in the vicinity of free surface, since the distribution of their neighboring particles is largely asymmetric as shown in Fig. 1.

In the present work, the fixed dummy particle method is adopted to present the wall boundary (Koshizuka et al. 1998), where three layers of boundary particles are fixed. In the multi-resolution simulation, only the differential models for the target wall particle $i$ are replaced using the following modified ones when the different-size particles are close to the target wall particle $i$.

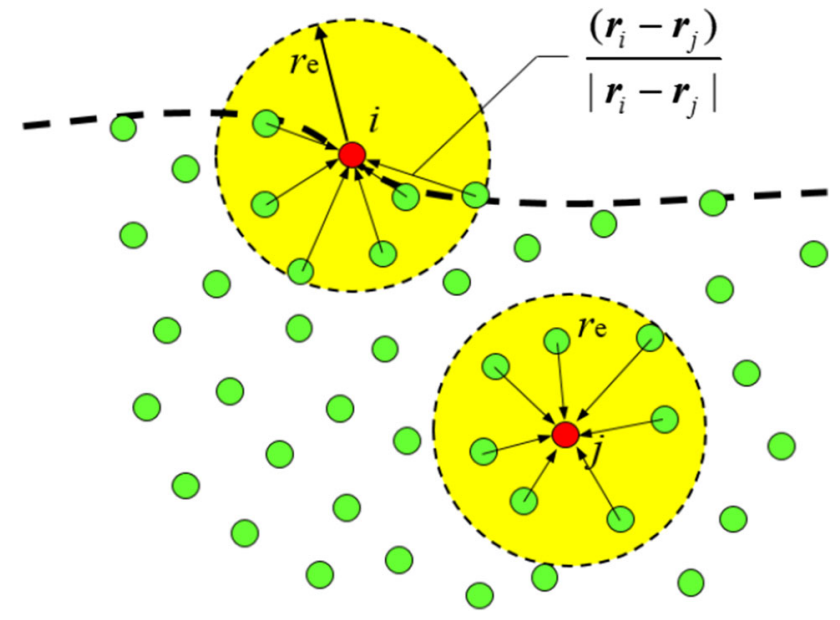

Fig. 1 Sketch of the asymmetry distribution of the neighboring particles

\subsection{Multi-resolution technique}

In the multi-resolution MPS, the cutoff radii for two neighboring particles are replaced by their averages to prevent the situation that the influence domains of these two particles cannot contain each other (Tanaka et al. 2009). In the present work, the influence radii of all particle interaction models are modified as in the following:

$r_{e}=\left(r_{e i}+r_{e j}\right) / 2$,

$r_{e \_ \text {lap }}=\left(r_{e i \_l a p}+r_{e j \_l a p}\right) / 2$,

where Eq. (16) is available for PND, gradient and divergence models, and Eq. (17) is used for the Laplacian model. Specifically, note that these modified cutoff radii have effects only for two different-size neighboring particles (hereinafter denoted as interface particles). These radii modifications are quite important for the conservation of the particle system, since it is the prerequisite for two neighboring particles following Newton's third law.

The same cutoff radii for two different-size neighboring particles can ensure that they can both be located in the influence domain of each other, but their interaction forces may not be equal since the particle size (or mass) is not involved in the standard MPS, which is quite different from the SPH method. Therefore, the differential models for interface particles in MPS should be modified by considering the particle size before multi-resolution simulations. In the present work, the modification for conservative pressure gradient (Eq. (5)) at particle $i$ can be given directly as

$$
\langle\nabla P\rangle_{i}=\frac{D}{n^{0}} \sum_{j \neq i} \frac{P_{j}+P_{i}}{\left|\mathbf{r}_{j}-\mathbf{r}_{i}\right|^{2}}\left(\mathbf{r}_{j}-\mathbf{r}_{i}\right) W\left(\left|\mathbf{r}_{j}-\mathbf{r}_{i}\right|\right) A_{j \rightarrow i},
$$


$A_{j \rightarrow i}=\frac{\frac{m_{j}}{L_{i} L_{j}}\left(L_{i}+L_{j}\right)}{\frac{m_{i}}{L_{i}}+\frac{m_{j}}{L_{j}}}$,

where $m$ and $L$ are the mass and diameter of the particle, respectively, and $A_{j \rightarrow i}$ can be considered as the modification for the kernel function in pressure gradient. Similar to Tanaka et al. (2009), PND and velocity divergence models are also employed in this modification.

In addition, the Laplacian model for interface particles is given as in the following by ignoring the nonlinear effect:

$$
\left\langle\nabla^{2} \phi\right\rangle_{i}=\frac{2 D}{n^{0}} \sum_{j \neq i}\left(\phi_{j}-\phi_{i}\right) W\left(\left|\mathbf{r}_{j}-\mathbf{r}_{i}\right|\right) \frac{\left(\frac{m_{j}}{L_{i}} / \lambda_{j}+\frac{m_{j}}{L_{j}} / \lambda_{i}\right)}{\frac{m_{i}}{L_{i}}+\frac{m_{j}}{L_{j}}} .
$$

\section{Motion computations}

In the present work, only the vertical displacement is allowed for the rigid body, and all the angular velocities are set as zero during the entire simulation. Therefore, the governing equations can simply be expressed as follows:

$M \frac{d^{2} \boldsymbol{r}_{G}}{\mathrm{~d} t}=M \mathbf{g}+\boldsymbol{F}_{\text {fluid-solid }}$,

where $M$ and $\boldsymbol{r}_{G}$ are the total mass and the gravity center of the rigid body, respectively, and $\boldsymbol{F}_{\text {fluid-solid }}$ is the hydrodynamic force acting on the rigid body. Furthermore, the force $\boldsymbol{F}_{\text {fluid-solid }}$ can be given as follows:

$\boldsymbol{F}_{\text {fluid-solid }}=-\iint_{S} P \mathrm{~d} S$,

where $P$ is the pressure on the wetted surface of the rigid body and $\mathrm{d} S$ is the area multiplied by the normal vector of the wetted surface of the rigid body.

\section{Numerical examples}

\subsection{D dam-break with obstacle}

In our previous work, the multi-resolution MPS was applied to 2D free-surface flows, including a hydrostatic problem and a 2D dam-break flow (Tang and Wan 2015). In the latter case, the recorded pressure between Uni-Fine and multi-MPS shows the overall tendency together with experimental data (Buchner 2002). In addition, the required CPU time by UniFine is nearly 2.5 times of that by multi-MPS, while particles in the latter is about a half of the former. In this section, a more complex dam-break model with an obstacle mounted in the bottom wall is also produced by both the uniform resolution and multi-resolution MPS methods to further validate the multi-resolution technique before discussing $3 \mathrm{D}$ applications in the following sections. The computational model is shown in Fig. 2. Three cases are considered and the main parameters are listed in Table 1. In the Multi-MPS case, the initial particle mass distribution is provided in Fig. 3, where the particles are colored by their masses.

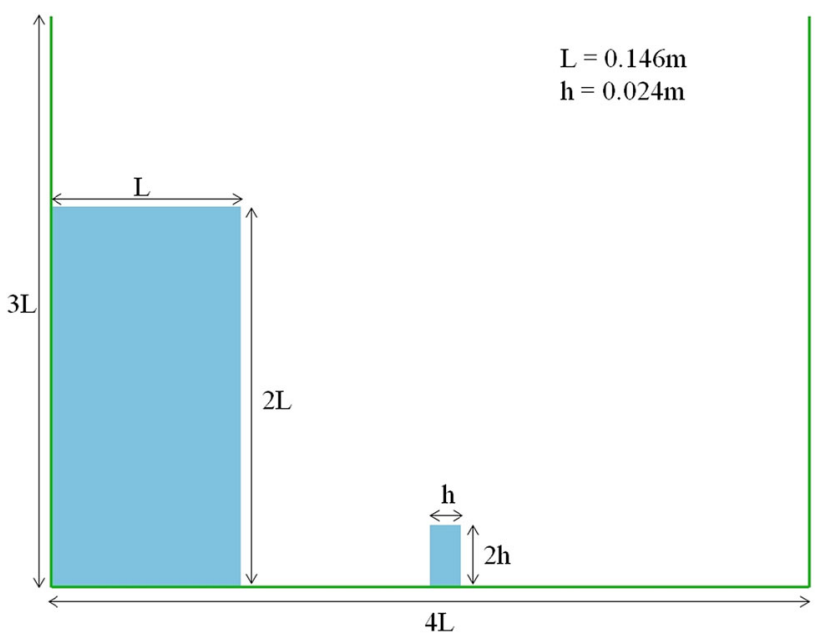

Fig. 2 A schematic sketch of the computational domain for 2D dambreak test

Table 1 Computational parameters used in the simulations

\begin{tabular}{lll}
\hline Cases & Initial particle space $(\mathrm{m})$ & Description \\
\hline Uni-Fine & 0.002 & Single resolution \\
Uni-Coarse & 0.008 & \\
Multi-MPS & $0.002 / 0.004 / 0.008$ & Multi-resolution \\
\hline
\end{tabular}

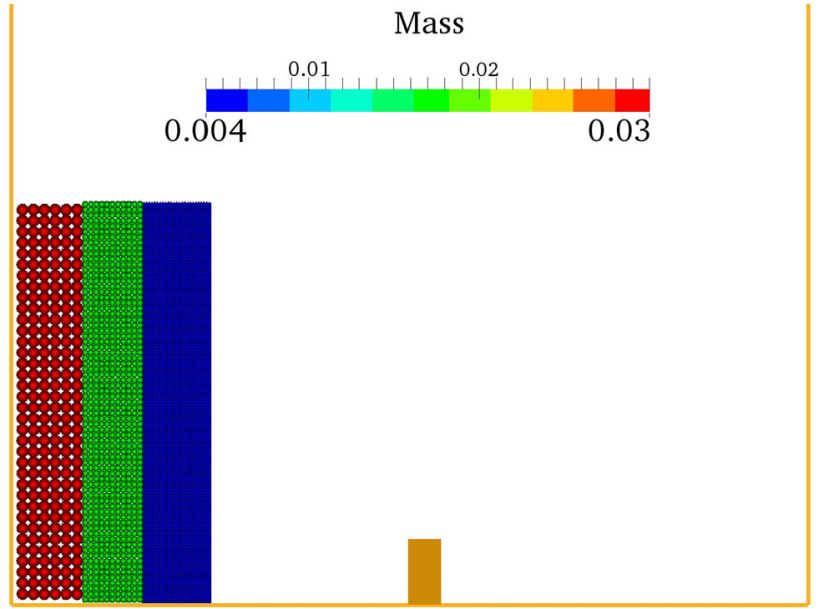

Fig. 3 Initial particle mass distribution for 2D dam-break problem 
Figures 4 and 5 show the comparison of free-surface deformations between the numerical results and experimental data (Koshizuka et al. 1995) at different instants of time. After the water column is released at the gate, the water front firstly moves along the bottom wall, and then impacts the obstacle. Later, a tongue jet can be observed clearly. Then, the jet moves toward the front right. It can be seen that the numerical methods can capture these phenomena which are also
Fig. 4 Snapshots of 2D

dam-break flows at $t=0.2 \mathrm{~s}$. a Exp. (Koshizuka et al. 1995), b Uni-Fine, $\mathbf{c}$ Uni-Coarse and d multi-MPS

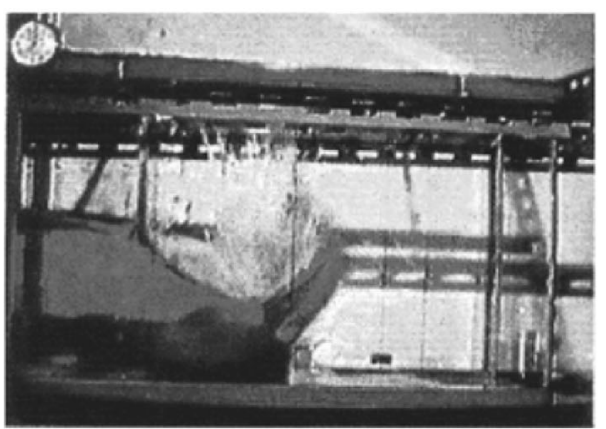

(a)

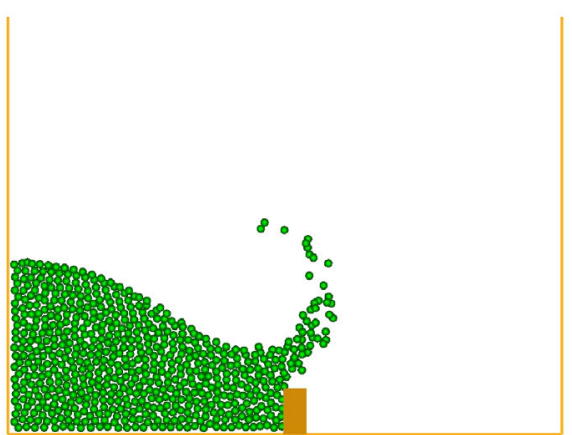

(c)

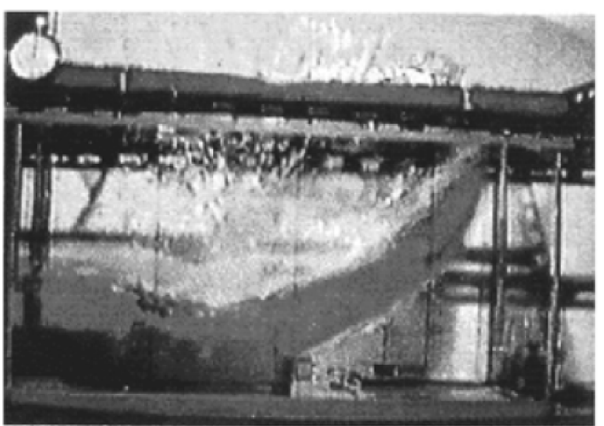

(a)

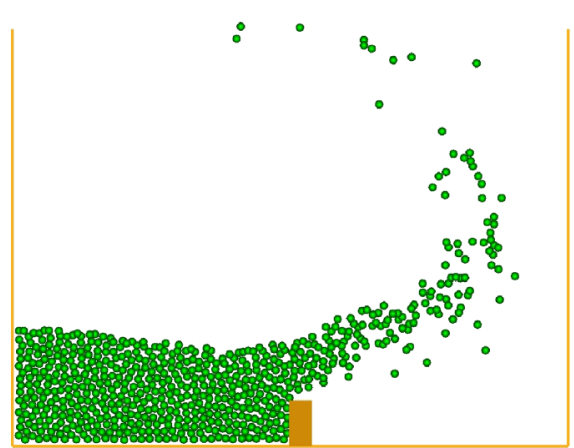

(c)

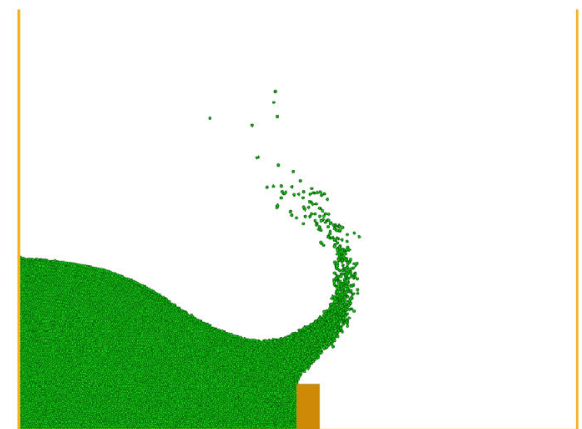

(b)

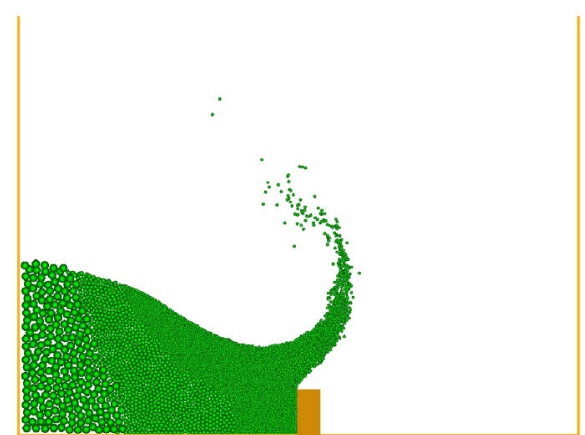

(d)

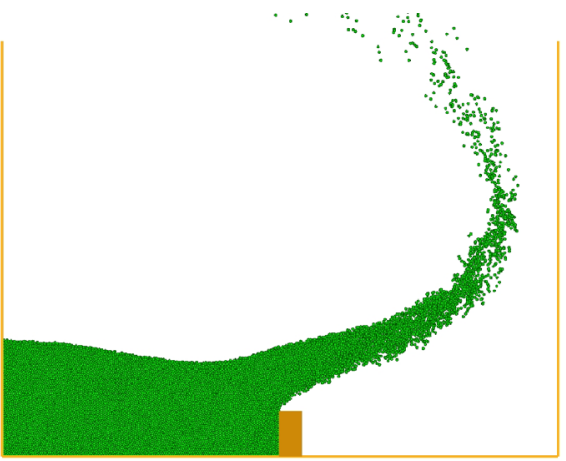

(b)

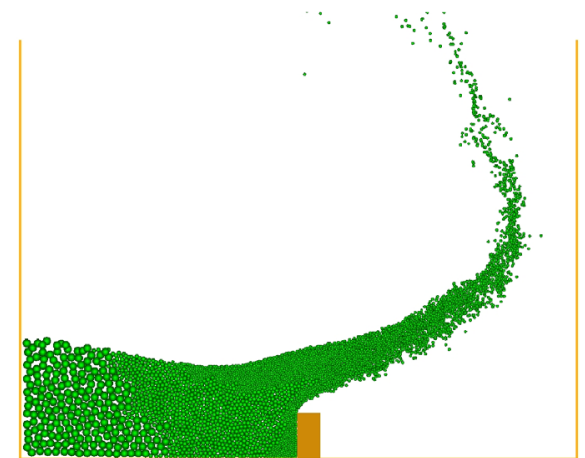

(d) 
Fig. 6 A schematic view of the computational domain for 3D dam-break problem (unit: $\mathrm{m}$ ). a geometry of dam-break and $\mathbf{b}$ positions of pressure probes

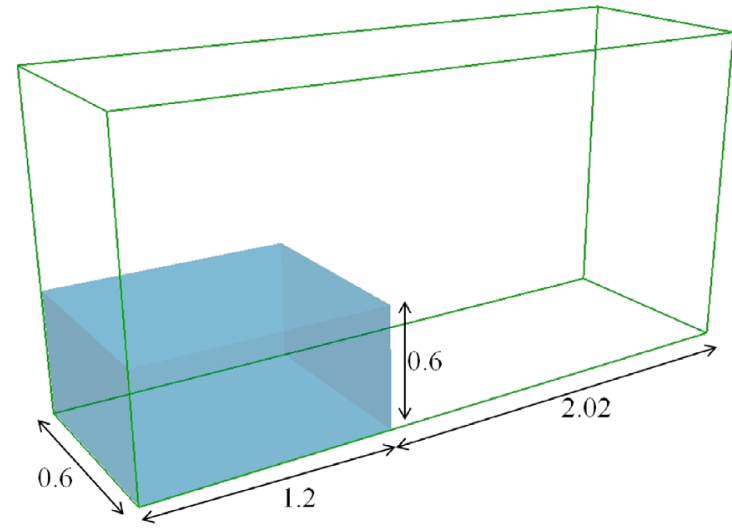

(a)

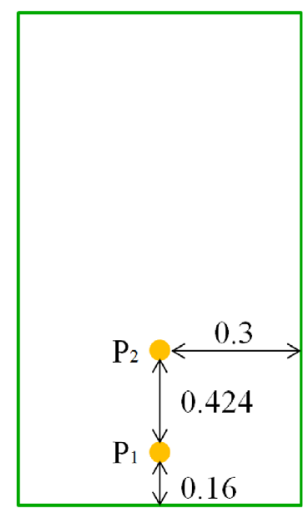

(b)

Table 2 Computational parameters used in the simulations

\begin{tabular}{lll}
\hline Case & Initial particle space $(\mathrm{m})$ & Description \\
\hline Multi-MPS & $0.01 / 0.02 / 0.04$ & Multi-resolution \\
\hline
\end{tabular}

quite similar to the experimental photographs (Koshizuka et al. 1995). However, the discrepancies can also be found among the three numerical simulations. The particle distributions by Multi-MPS is much more integrated than that by Uni-Coarse, but is close to that by Uni-Fine, especially before the jet impacting the right wall. In addition, the shapes of free surface by Multi-MPS and Uni-Fine are also much clearer than that by Uni-Coarse after the water front impacts the obstacle. Furthermore, the CPU time by Multi-MPS is nearly $30 \%$ of that by Uni-Fine in this case. Therefore, only the multi-resolution MPS is employed to carry out 3D cases in the following sections.

\subsection{D dam-break flow}

Dam-break is a violent free-surface flow accompanied by a complex phenomena including the overturn of free surface, the jet impacting water and liquid splashing. In this section, a $3 \mathrm{D}$ dam-break flow is simulated by the multi-resolution MPS method. A schematic view of the computational domain is shown in Fig. 6a, and two pressure probes are placed on the right wall as shown in Fig. 6b. In this case, three kinds of particle size are selected and the corresponding parameters are listed in Table 2. In addition, the initial particle mass distribution is shown in Fig. 7.

Some snapshots of the numerical flow fields are shown in Fig. 8, where particles are colored employing their pressures. Similar to the previous case, the water front first moves along the bottom of the tank and then impacts the right wall, resulting in water splashing as shown in Fig. 8b. Then, the evolution of impact pressure at two pressure probes $P_{1}$ and $P_{2}$ are both illustrated in Fig. 9, where the multi-resolution

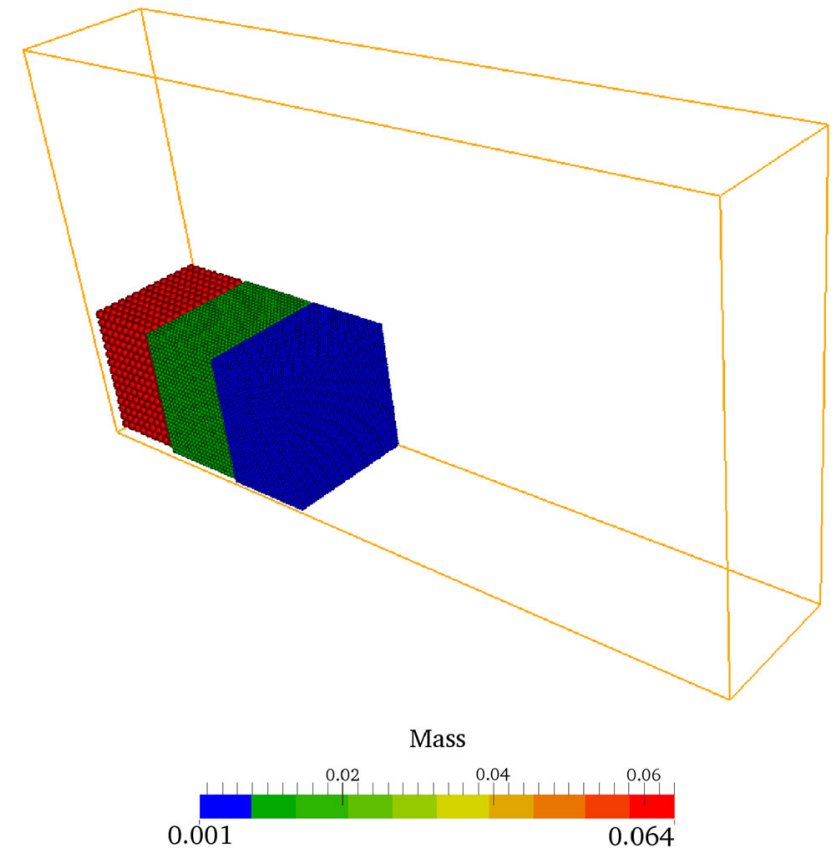

Fig. 7 Initial particle mass distribution for 3D dam-break problem

MPS results are compared with the experimental data (Buchner 2002). The overall tendency of pressure variations at both $P_{1}$ and $P_{2}$ by the numerical method is in agreement with the experimental data (Buchner 2002). The pressure peak at $\mathrm{P}_{1}$ is also quite close to that in the experiments (Buchner 2002), which occurs when the overturned free surface impacts the underlying water. However, the phase difference between the numerical results and experimental data can also be observed at $P_{1}$. The main reason behind this is that only the one-phase model is adopted in the present work. After the jet impacts the underlying water, a cavitation is formed and air can also be entrapped in the cavitation. In this situation, the air effect cannot be ignored and a two-phase model may improve the results, which had been pointed out by Lind et al. (2016). 


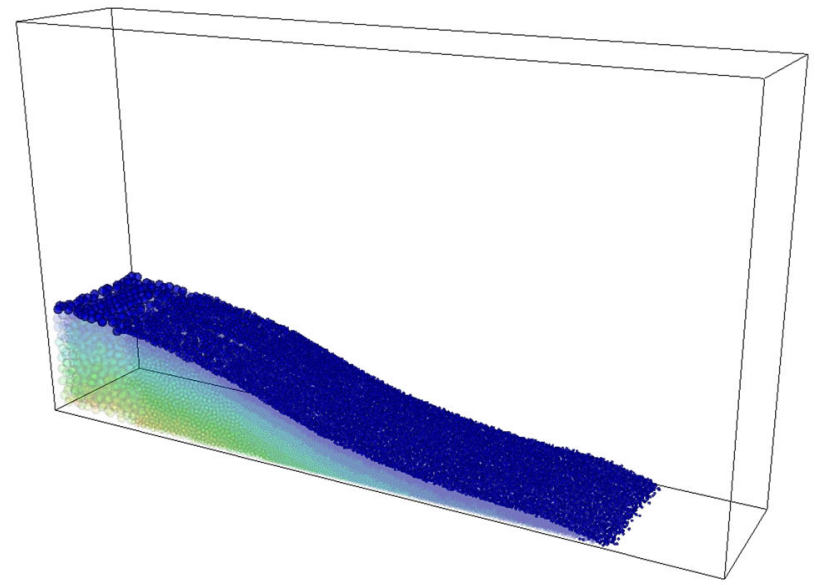

(a)

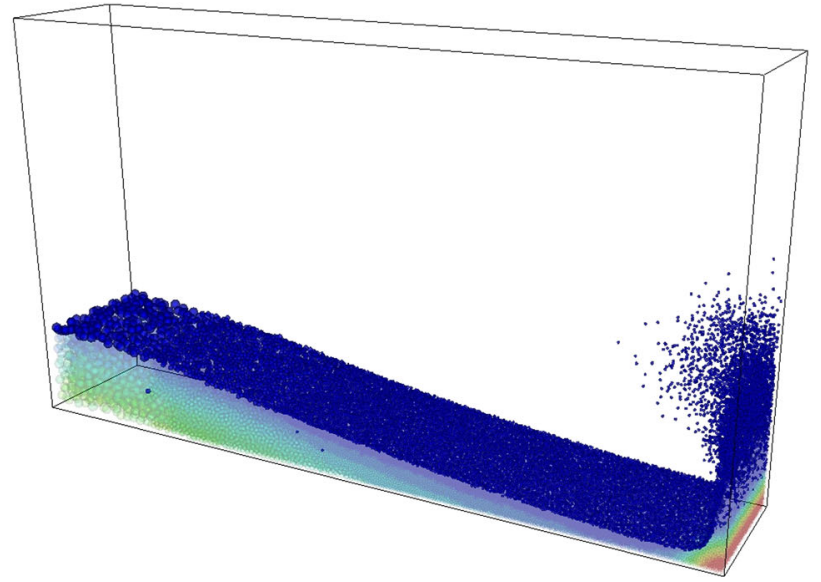

(b)

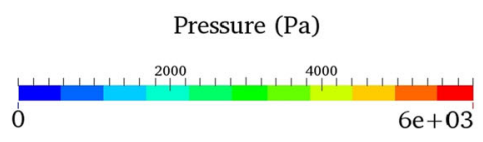

Fig. 8 Snapshots of the 3D dam-break problem using multi-resolution MPS at different instants of time. a $t=0.50 \mathrm{~s}$ and $\mathbf{b} 0.76 \mathrm{~s}$

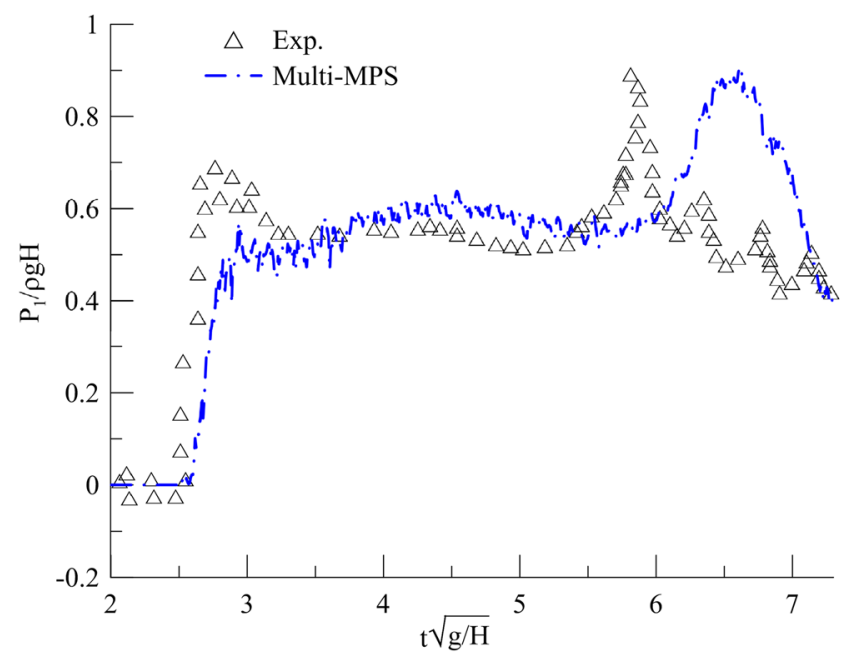

(a)

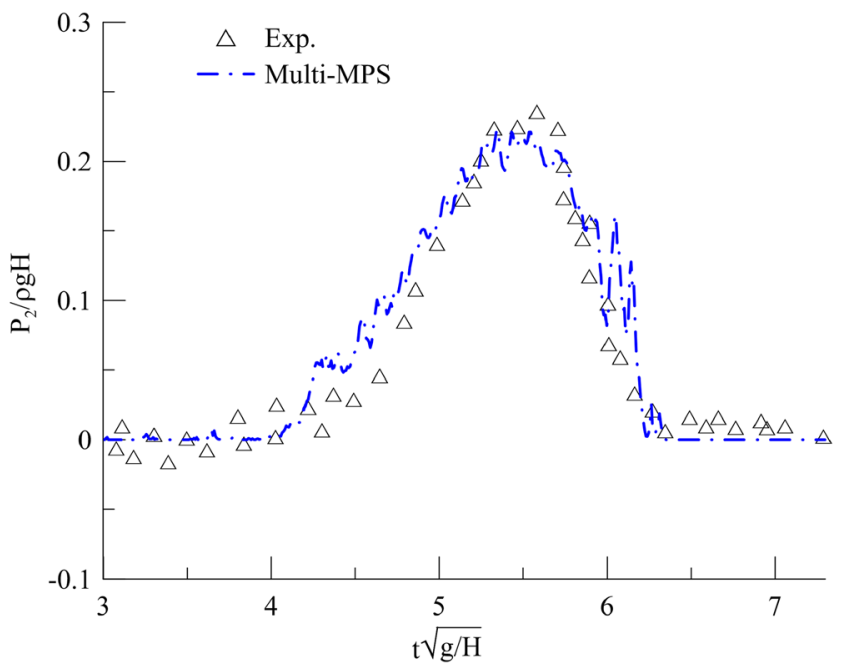

(b)

Fig. 9 The predicted dimensionless pressure at two pressure probes between experimental data (Buchner 2002) and the present results. a $P_{1}$ and b $P_{2}$

\subsection{D water entry of a neutral buoyant circular cylinder}

In this section, a 3D water entry of the cylinder is carried out by multi-resolution MPS, where the corresponding experiment was carried out by Greenhow and Lin (1983). A schematic view of the computational domain can be seen in Fig. 10. The center of the cylinder with diameter $0.11 \mathrm{~m}$ is $0.5 \mathrm{~m}$ from the water level, and its density is $1000 \mathrm{~kg} / \mathrm{m}^{3}$. The cylinder is released at $t=0 \mathrm{~s}$ and only allowed to move in the vertical direction. As reported by Sun and Faltinsen
(2006), the cylinder begins to impact the underwater at about $t_{0}=0.301 \mathrm{~s}$ with an entry speed of $2.955 \mathrm{~m} / \mathrm{s}$. In our numerical experiments, the computational domain is discretized by three kinds of particle sizes listed in Table 3 and the initial particle mass distribution is shown in Fig. 11, where the zone close to the impact area is distributed with the finest particles and one far from the impact area is represented by the coarsest particles. In this case, the kinematic viscosity of water is set to be $1.01 \times 10^{-6} \mathrm{~m}^{2} / \mathrm{s}$.

Figure 12 shows the snapshots of free-surface deformations and pressure fields during the 3D cylinder entering 


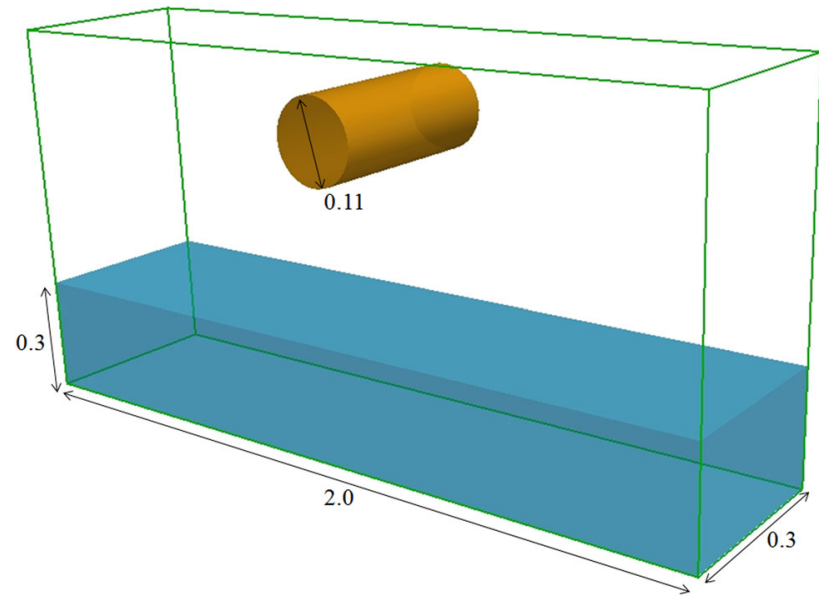

Fig. 10 A schematic view of the computational domain for 3D water entry

Table 3 Computational parameters used in the simulations

\begin{tabular}{lll}
\hline Case & Initial particle space $(\mathrm{m})$ & Description \\
\hline Multi-MPS & $0.005 / 0.01 / 0.02$ & Multi resolution
\end{tabular}
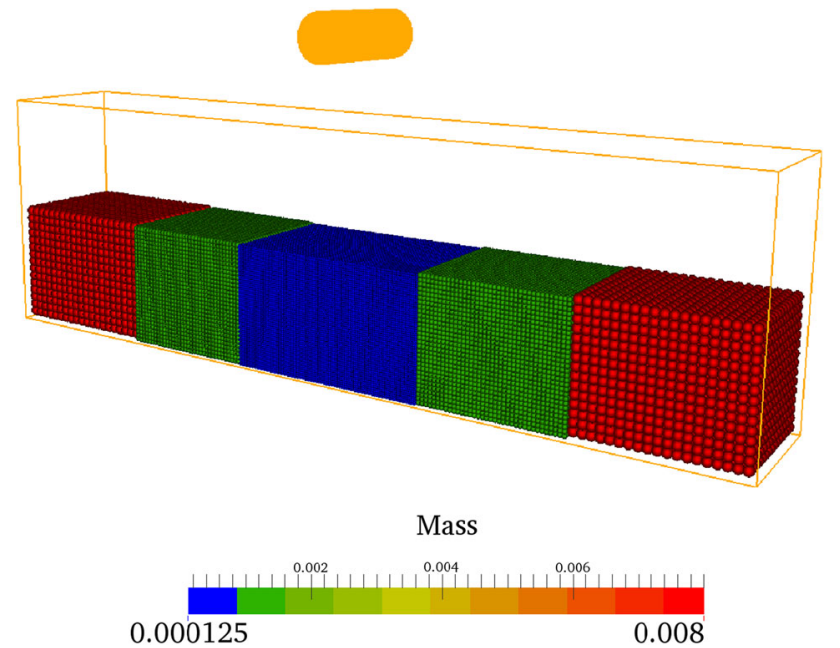

Fig. 11 Initial particle distribution for 3D water entry

water at different instants of time, where the particles are colored by their pressures. From these figures, it can be observed that the flow fields are quite violent after the $3 \mathrm{D}$ cylinder enters the water. When the cylinder impacts the underlying water, a high pressure area under the cylinder is generated. It is expected that water exerts a large force on the 3D cylinder. As the cylinder continues to move down, a jet is formed on each side of the cylinder and the splashing is also very obvious. In addition, the area of high pressure region under the cylinder decreases.
Furthermore, the comparison of depth of penetration between the numerical results and referenced data is also shown in Fig. 13, where the triangle indicates the experimental data (Greenhow and Lin 1983) and the green line represents the BEM result (Sun and Faltinsen 2006). Here, the time axis represents the relative time from the initial impact one $t_{0}$. It can be seen that the overall tendency of penetration depth by multi-resolution simulation is in agreement with experimental data (Greenhow and Lin 1983) and BEM result (Sun and Faltinsen 2006). From this figure, it can be found that the cylinder does not touch the bottom wall at $t=0.2 \mathrm{~s}$, while it reaches the bottom wall in the experiments. That is to say, the penetration speed in the simulations is slower than that in the experiments. The main reason may be that the air effect is not considered in this paper. In this case, the entry speed reaches nearly $3.0 \mathrm{~m} / \mathrm{s}$ and the flow is quite violent and complex; there may not be enough time for air to escape from the surface of the 3D cylinder.

\section{Conclusions}

In the present work, 3D violent free-surface flows are carried out numerically. Firstly, a 2D dam-break flow is performed to validate the performance of the multi-resolution MPS. The comparisons of the shape of the free surface among uniform resolution and multi-resolution numerical results and experimental photographs (Koshizuka et al. 1995) show that Multi-MPS can portray more integrated free surface than that by Uni-Coarse, but is similar to Uni-Fine. The profile of the jet by the former is also clearer than that by the latter. In addition, the CPU time by Multi-MPS is less than that by Uni-Fine. Then, the multi-resolution MPS is applied for 3D free-surface flows. In the dam-break case, the recorded pressures at two probes are compared with the experimental data (Buchner 2002). The overall tendency of pressure variation and pressure peak by multi-MPS is in good agreement with experiments except that the phase of pressure peak at $P_{1}$ lags the experiments, due to the one-phase model adopted in the present work. In the 3D water entry case, a cylinder impacts the underlying water and a jet forms on each side of the cylinder. When the cylinder further goes down, complex phenomena such as largely free-surface deformation and splashing can also be observed clearly. The penetration depth of the cylinder is also very close to experimental data (Greenhow and Lin 1983). The discrepancy between numerical results and experiment may be caused by the one-phase model used in this work. 


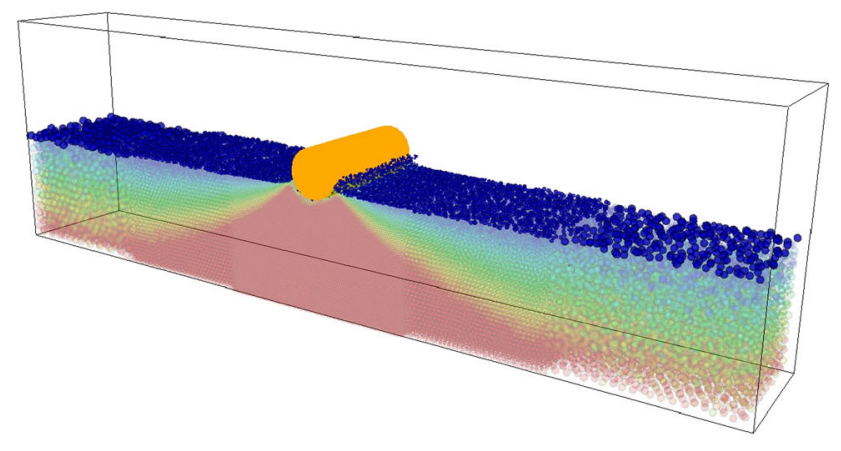

(a)

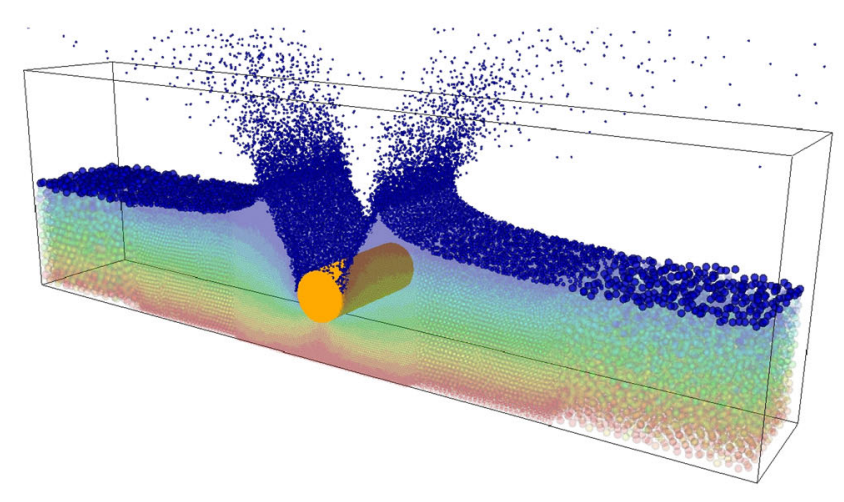

(c)

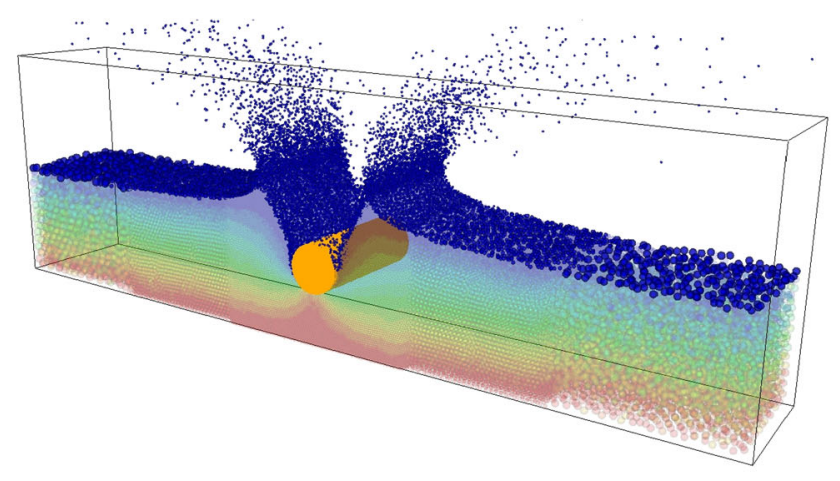

(b)

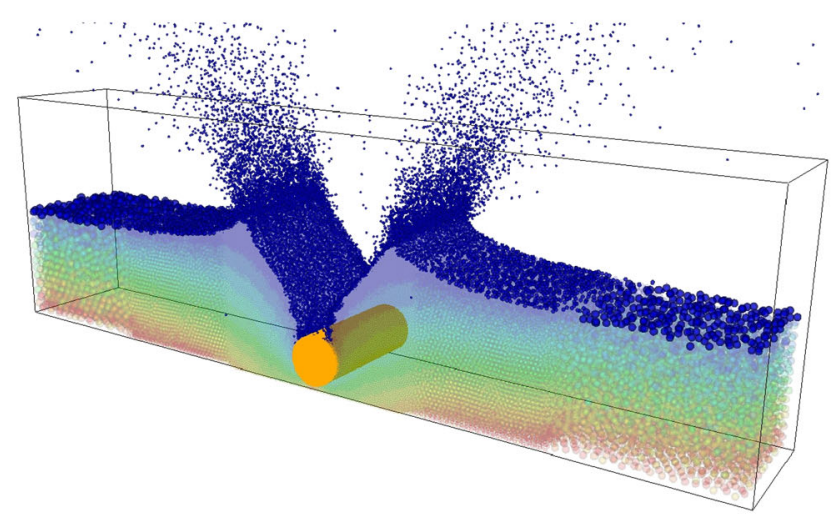

(d)

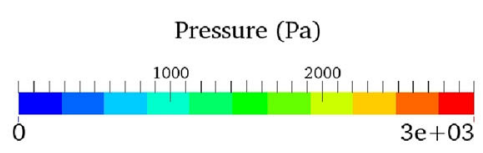

Fig. 12 Snapshots of 3D water entry flows using multi-resolution MPS at different instants of time. a $t=0.31 \mathrm{~s}, \mathbf{b} t=0.39 \mathrm{~s}, \mathbf{c} t=0.41 \mathrm{~s}$ and $\mathbf{d} t=0.50 \mathrm{~s}$

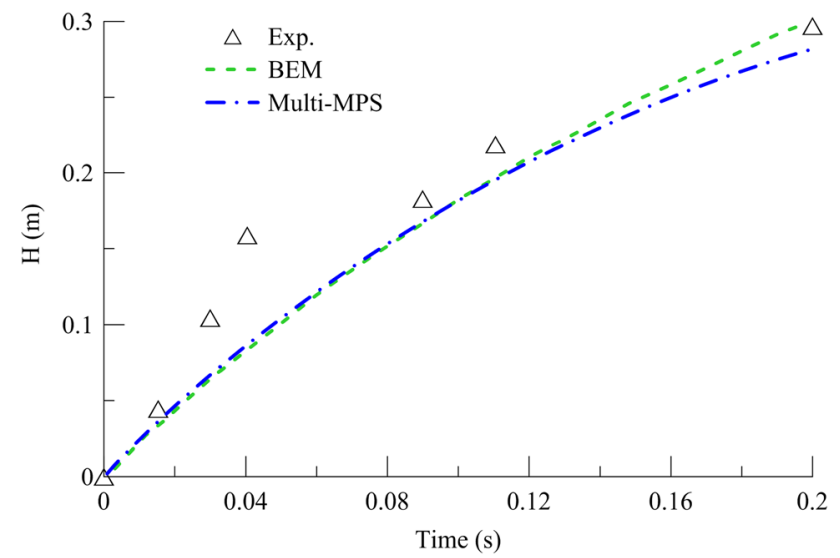

Fig. 13 Depth of penetration of the circular cylinder among experimental data (Greenhow and Lin 1983), BEM result (Sun and Faltinsen 2006) and the present results
However, improvements should be further made. For the long time simulations such as violent liquid sloshing flows, it is hard to ensure the situation where the initial refine region is always occupied by high-resolution particles, and differentsize particles may mix randomly since no particle splitting and coalescing algorithms are implemented in the present work. Nevertheless, particle splitting and coalescing may lead to pressure discontinuity. In the future, these problems should be further investigated.

Acknowledgments This work was supported by the National Natural Science Foundation of China (51379125, 51490675, 11432009, 51579145, 11272120), Chang Jiang Scholars Program (T2014099), Program for Professor of Special Appointment (Eastern Scholar) at Shanghai Institutions of Higher Learning (2013022) and Innovative Special Project of Numerical Tank of Ministry of Industry and Information Technology of China (2016-23/09), to which the authors are most grateful. 


\section{References}

Barcarolo DA, Le Touzé D, Oger G, de Vuyst F (2014) Adaptive particle refinement and derefinement applied to the smoothed particle hydrodynamics method. J Comput Phys 273:640-657

Buchner B (2002) Green water on ship-type offshore structures. PhD Thesis, Technische Universiteit Delft

Chen R, Tian W, Su GH, Qiu S, Ishiwatari Y, Oka Y (2010) Numerical investigation on bubble dynamics during flow boiling using moving particle semi-implicit method. Nucl Eng Des 240(11):3830 3840

Duan G, Chen B (2015) Comparison of parallel solvers for moving particle semi-Implicit method. Eng Comput 32(3):834-862

Gotoh H, Khayyer A, Ikari H, Hori C (2009) Refined reproduction of a plunging breaking wave and resultant splash-up by 3D-CMPS method. In: Proceedings of the Nineteenth International Offshore and Polar Engineering Conference, pp 518-524

Greenhow M, Lin W-M (1983) Non-linear free surface effects experiments and theory. Massachusetts Institute of Technology, Cambridge

Heo S, Koshizuka S, Oka Y (2002) Numerical analysis of boiling on high heat-flux and high subcooling condition using MPS-MAFL. Int J Heat Mass Transf 45(13):2633-2642

Hori C, Gotoh H, Ikari H, Khayyer A (2011) GPU-acceleration for moving particle semi-implicit method. Comput Fluids 51:174-183

Iribe T, Fujisawa T, Koshizuka S (2010) Reduction of communication in parallel computing of particlem method for flow simulation of seaside areas. Coast Eng J 52(4):287-304

Kakuda K, Nagashima T, Hayashi Y, Obara S, Toyotani J, Katsurada N, Matsuda S (2012) Particle-based fluid flow simulations on GPGPU using CUDA. Comput Model Eng Sci 88(1):17-28

Kakuda K, Nagashima T, Hayashi Y, Obara S, Toyotani J, Miura S, Katsurada N, Higuchi S, Matsuda S (2013) Three dimensional fluid flow simulations using GPU-based particle method. Comput Model Eng Sci 93(5):363-376

Khayyer A, Gotoh H, Shao S (2009) Enhanced predictions of wave impact pressure by improved incompressible SPH methods. Appl Ocean Res 31(2):111-131

Khayyer A, Gotoh H (2008) Development of CMPS method for accurate water-surface tracking in breaking waves. Coast Eng J 50(2):179_ 207

Khayyer A, Gotoh H (2013) Enhancement of performance and stability of MPS mesh-free particle method for multiphase flows characterized by high density ratios. J Comput Phys 242:211-233

Koshizuka S, Oka Y, Tamako H(1995) A particle method for calculating splashing of incompressible viscous fluid. Int Conf Math Comput Reactor Phys Environ Anal 2:1514-1521

Koshizuka S, Nobe A, Oka Y (1998) Numerical analysis of breaking waves using the moving particle semi-implicit method. Int J Numer Meth Fluids 26(7):751-769

Koshizuka S, Oka Y (1996) Moving-particle semi-implicit method for fragmentation of incompressible fluid. Nucl Sci Eng 123(3):421434

Lee B-H, Park J-C, Kim M-H, Hwang S-C (2011) Step-by-step improvement of MPS method in simulating violent free-surface motions and impact-loads. Comput Methods Appl Mech Eng 200:11131125

Lind SJ, Stansby PK, Rogers BD (2016) Incompressiblecompressible flows with a transient discontinuous interface using smoothed particle hydrodynamics (SPH). J Comput Phys 309: 129-147

Marrone S, Colagrossi A, Antuono M, Lugni C, Tulin MP (2011) A $2 \mathrm{D}+\mathrm{t} \mathrm{SPH}$ model to study the breaking wave pattern generated by fast ships. J Fluids Struct 27(8):1199-1215
Oger G, Doring M, Alessandrini B, Ferrant P (2006) Two-dimensional $\mathrm{SPH}$ simulations of wedge water entries. J Comput Phys 213(2):803-822

Omidvar P, Stansby PK, Rogers BD (2013) SPH for 3D floating bodies using variable mass particle distribution. Int J Numer Meth Fluids 72(4):427-452

Ovaysi S, Piri M (2012) Multi-GPU acceleration of direct pore-scale modeling of fluid flow in natural porous media. Comput Phys Commun 183(9):1890-1898

Shakibaeinia A, Jin Y-C (2011) A mesh-free particle model for simulation of mobile-bed dam break. Adv Water Resour 34(6):794-807

Shakibaeinia A, Jin Y-C (2012) MPS mesh-free particle method for multiphase flows. Comput Methods Appl Mech Eng 229-232:1326

Shibata K, Masaie I, Kondo M, Murotani K, Koshizuka S (2015) Improved pressure calculation for the moving particle semiimplicit method. Comput Particle Mech 2(1):91-108

Shibata K, Koshizuka S, Tamai T (2012) Overlapping particle technique and application to green water on deck. In: 2nd International Conference on Violent Flows, pp 106-111

Sun H, Faltinsen OM (2006) Water impact of horizontal circular cylinders and cylindrical shells. Appl Ocean Res 28(5):299-311

Tamai T, Koshizuka S (2014) Least squares moving particle semiimplicit method. Comput Particle Mech 1(3):277-305

Tanaka M, Masunaga T (2010) Stabilization and smoothing of pressure in MPS method by quasi-compressibility. J Comput Phys 229(11):4279-4290

Tanaka M, Masunaga T, Nakagawa Y (2009) Multi-resolution MPS method. Transactions of JSCES

Tang Z, Zhang Y, Wan DC (2016a) Numerical simulation of 3D free surface flows by overlapping MPS. J Hydrodyn 28(2):306-312

Tang Z, Wan DC (2015) Numerical simulation of impinging jet flows by modified MPS method. Eng Comput 32(4):1153-1171

Tang Z, Zhang Y, Wan DC (2016b) Multi-resolution MPS method for free surface flows. Int J Comput Methods 13(4):1641018. doi:10. 1142/S0219876216410188

Tian W, Ishiwatari Y, Ikejiri S, Yamakawa M, Oka Y (2009) Numerical simulation on void bubble dynamics using moving particle semiimplicit method. Nucl Eng Des 239(11):2382-2390

Vacondio R, Rogers BD, Stansby PK (2012) Accurate particle splitting for smoothed particle hydrodynamics in shallow water with shock capturing. Int J Numer Meth Fluids 69(8):1377-1410

Vacondio R, Rogers BD, Stansby PK, Mignosa P, Feldman J (2013) Variable resolution for SPH: a dynamic particle coalescing and splitting scheme. Comput Methods Appl Mech Eng 256:132-148

Yoon HY, Koshizuka S, Oka Y (1999) A particle-gridless hybrid method for incompressible flows. Int J Numer Meth Fluids 30(4):407-424

Zhang Y, Wan DC, Hino T (2014a) Application of MPS method in liquid sloshing. Ocean Eng 32(4):24-32

Zhang Y, Wan DC, Hino T (2014b) Comparative study of MPS method and level-set method for sloshing flows. J Hydrodyn 26(4):577585

Zhang Y, Tang Z, Wan DC (2016) Numerical investigations of waves interacting with free rolling body by modified MPS method. Int J Comput Methods 13(4):1641013. doi:10.1142/ S0219876216410139

Zhang Y, Wan DC (2012) Numerical simulation of liquid sloshing in low-filling tank by MPS. Chin J Hydrodyn 27:100-107

Zhang Y, Wang X, Tang Z, Wan DC (2013) Numerical simulation of green water incidents based on parallel MPS method. In: Proceedings of the Twenty-third International Offshore and Polar Engineering, pp 931-938

Zhu X, Cheng L, Lu L, Teng B (2011) Implementation of the moving particle semi-implicit method on GPU. Sci China Phys Mech Astron 54(3):523-532 